ARTICLE INFO.

Article History

Received: 8 January 2021

Received in Revised form: 14 January 2021

Accepted: 2 February 2021

Keywords

Management

Accountant

\section{Intelligent Traffic Management system based on IoT Challenges and Applications}

Samah A. Gamel, and Ahmed I. Saleh, and Hesham A. Ali

Computer Engineering and Control Systems Dept. faculty of engineering

Mansoura University, Egypt

\begin{abstract}
Analytics of Big data and the Internet of Things (IoT) are critical for developing intelligent transportation systems in smart cities. IoT has the ability to mitigate the urbanization pressures, provide new experiences and make everyday life easier and safer for city residents. In this article, we look at the uses and applications of IoT in decreasing traffic congestion problems and analyze the challenges of implementing IoT-enabled systems for traffic management.
\end{abstract}

\title{
Keywords
}

IoT; Intelligent Transport Systems; Real-Time Traffic Control Systems; Smart Cities; Non-intrusive Sensors

\section{Introduction}

According to the UN forecasts, the urban population in the globe is expected to double by 2050 to reach 6.7 million inhabitants. As the number of towns and cities rises, new opportunities and challenges are appearing [1]. Municipalities are turning to the Internet of Things (IoT) to help prevent environmental deterioration, minimize sanitary issues, reduce traffic congestion, and prevent urban crime. With the technological development, sensors are developed with connection to the internet and allow things to communicate, learn and carry out specific activities. This Internet connection of things is referred to as the Internet of Things (IoT), which autonomously uses the given information and executes the duties as intended. Devices that can monitor or control the physical parameter of the environment are the true physical interface to the world [2]. In nearly all areas of health care, home automation, transportation, and industry, IoT devices are employed. This has increased cost efficiency and decreased the company's massive workload. At this time, companies incorporate IoT into nearly all links to the internet and could to the internet. IoT offers 
enormous potential to employ big data analytics in the transformation of cities built on infrastructure.

The sustainability and intelligence of the smart city idea depend on the technologies chosen to enhance the quality of life of the inhabitants. Smart-city management is a significant part of smart-city efforts that allow improved decision-making technology [3][4]. The public value created by the intelligent services offered [5] is one of the fundamental aspects of the smart city governance system. The government must focus on many areas of intelligentsia solutions like intelligent health services, intelligent construction management, intelligent traffic management, intelligent parking, intelligent transportation, etc. This will result in a great value for the provided services. The physical infrastructures in smart cities are equipped with intelligent sensors and devices, which periodically generate big data from various locations. These data are used to develop intelligence for all aspects of the smart city [6]. Ultimately, IoT and intelligence are used to enhance social life and economic activities.

The Smart city combines and exchanges diverse information systems that are gathered by smart infrastructure, people, and things to produce new insight and deliver omnipresent services that enable the public to access city information, to move around catastrophes. IoT is the design of various instruments in combination to function as an autonomous and intelligent agent to do the activities simply and efficiently. An intelligent city combined with IoT can enhance the infrastructure of a city. Applications include healthcare, architecture, environmental services, water treatment, level of radiation, leakage of water, location of items, intelligent parking, intelligent packaging, access control perimeter, smart lighting, smart streets [7] as illustrated in Figure 1.

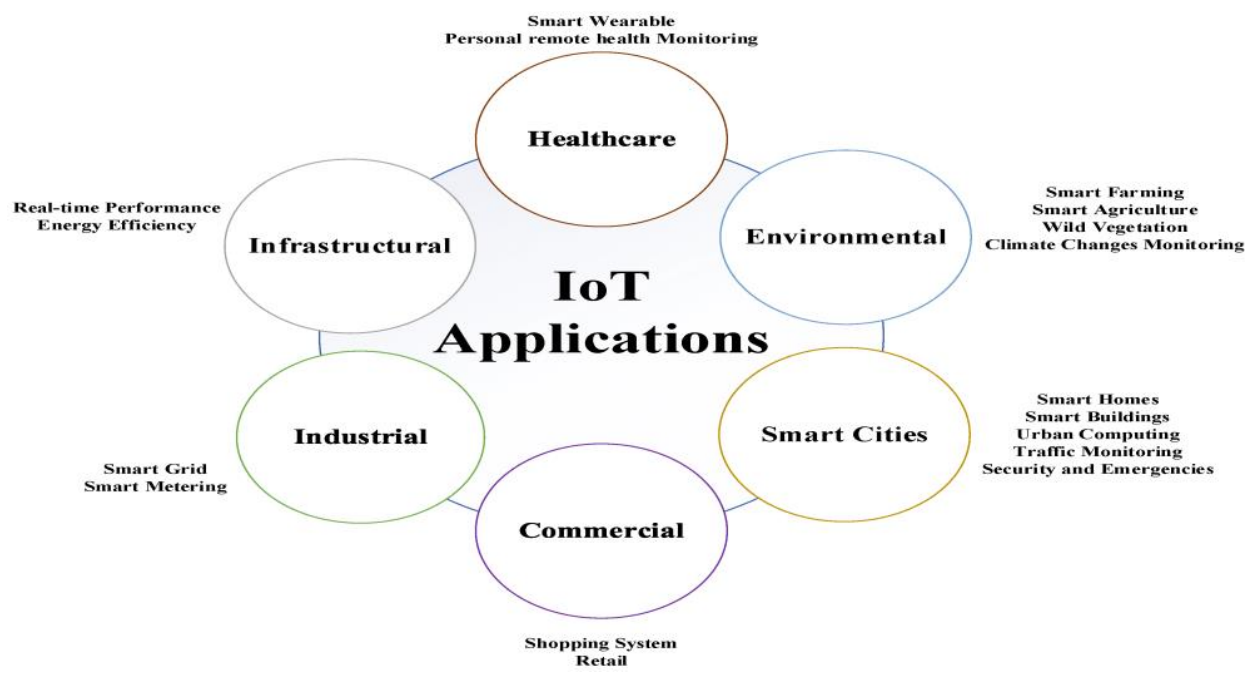

Figure 1: IoT Applications on smart cities 


\section{Smart Traffic Management System based on IoT (TMS)}

One of the essential components of the smart city is the smart infrastructure of the traffic system. As, with the growth of city development, traffic congestion increases and became a critical issue. Intelligent transportation systems with integrated components such as highway management adaptive traffic signal controls, roadside units, and emergency management services are all examples of smart traffic management [8]. These systems collect real-time traffic data and take the required measures to prevent or reduce any social issues that arise as a result of traffic congestion [9]. For example, people will be able to use real-time traffic maps to help them choose the best route to save effort and time.

Based on sensor data from monitoring equipment deployed on highways and urban roads, popular mobile applications such as Google Maps and Apple Maps reliably forecast traffic congestion for urban roads and highways [10]. These applications vendors cooperate in the collection of traffic information with different carriers. The majority of traffic monitoring equipment is installed on metropolitan roadways by transportation authorities, thus such application providers (for example, Google application programming interface) provide traffic congestion updates. In addition, such programs also employ location-based crowdsourcing [11] to enhance the forecast of traffic density. It expects intelligent technologies to be updated in real-time on the road in the car or on any smart mobile equipment with the driver. The problem is that consumers need intelligent gadgets to access such apps and most services are restricted to metropolitan roadways.

Figure 2 illustrates the fields of IoT in the traffic system. The traffic patterns on metropolitan roads and motorways differ from those on collector roads. Collector roads contain bicycles, walkers, motorcycles, and other vehicles; the patterns of road transport are therefore distinct from those of roadways. Along with urban roadways, real-time monitoring of collector roads is critical for improving overall city transportation. Real-time traffic congestion updates [12], as well as warnings from traffic controllers on non-recurring traffic incidents such as spilled cargoes, accidents, emergency services, VIP visits, or any other unusual occurrences, would assist collector road drivers in making decisions. During peak hours, for example, closed institutions such as colleges and hospitals experience significant traffic congestion. These campuses will have multiple collector road segments of varying lengths connecting to various entry/exit locations.

The real-time traffic updates of roads that connect to exit locations may assist the motorist in picking the best route from his present position. Drivers like to be aware of the congestion status of upcoming junctions in order to plan ahead of 
time and save time on the road by using other routes. The challenge here is how to give real-time road congestion information to drivers in the absence of such smart sensors with them or within the vehicles, which is the true impetus for this research [13].

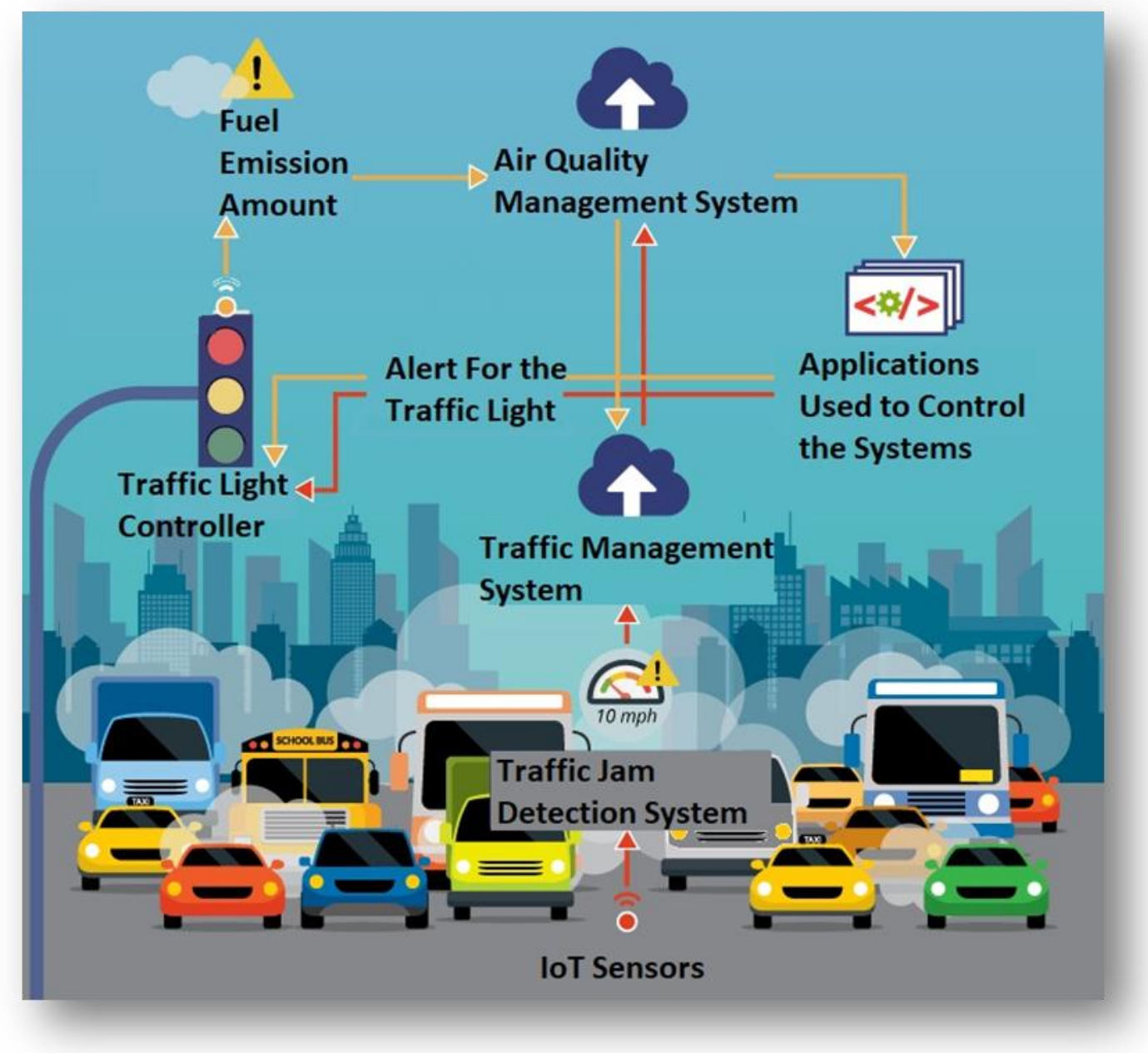

Figure2: Applications of IoT in the m n

Research in smart transport systems recently shown that the IoT paradigm may play a significant role in the management of traffic through the connection of physical objects over the internet for information sharing, tracker, and traffic tracking [14]. Global positioning systems, test vehicles, sensors, and cars for communicating infrastructure are some means of gathering road data in real-time. Acoustical and magnet sensors are efficient, cost-effective and the latest vehicle surveillance systems are the most popular [15]. The real-time traffic data from different sources can be utilized for traffic congestion prediction and management. In particular, smart mobile devices [16] offer several current alternatives for realtime traffic updates of metropolitan roadways, while collector roads have received 
little attention. Therefore, this research focus on IoT-based systems challenges to overcome and enhance the real-time traffic updates through different sensors.

\section{IoT Challenges on Traffic Management System on Smart City}

Following are the problems that must be solved in the implementation of IoT for traffic management, taking into account the steps that intelligent transportation systems adopt.:

1. Accuracy: The sensors may be at risk, particularly in unfavorable weather, high-traffic circumstances or accident situations, or technological failures, to supply inaccurate information.

2. Security and privacy: When IoT is used in intelligent transportation systems, concerns and challenges relating to privacy and system security exist. According to the research in [14], about $70 \%$ of IoT devices in a smart city may be in danger of infringement because of bugs and vulnerabilities such as weak authorization systems, firewall absence, or detection protection systems. Sensors have a restricted power volume, resulting in a compromise in security functions such as poorly encrypted protocols for communication [14].

3. Overload and integration in the network: It is quite complicated for Advanced Transport Management Systems to provide an overview of each driver's traffic which might overload the network resulting in a failure. Another key difficulty is the integration of data into the traffic management system based on IoT, as each detector needs to be individually measured and standardized.

4. Inefficiency in terms of traffic risks: As most IoT-dependent Advanced Transport Management Systems cannot identify traffic risks in realtime, this results in a system working failure as it is useless at that time and takes hours to update the system with this information.

5. Interoperability: Interoperability is the ability of two devices to interact and is a significant challenge in Traffic Management Systems. Since it takes time for data to be fetched during the Vehicle to Vehicle (V2V) interaction, the connection might be disrupted and data loss [15].

\section{CONCLUSION}

In this paper, We provided an overview of IoT, intelligent cities, and IoT use in traffic congestion management. We also addressed major difficulties to be dealt with when using IoT technologies to reduce traffic congestion 


\section{References}

1. https://www.un.org/en/sections/issues-depth/population/

2. Kandris, D., Nakas, C., Vomvas, D., \& Koulouras, G. (2020). Applications of wireless sensor networks: an up-to-date survey. Applied System Innovation, 3(1), 14.

3. Tran Thi Hoang, G., Dupont, L., \& Camargo, M. (2019). Application of decision-making methods in smart city projects: a systematic literature review. Smart Cities, 2(3), 433-452.

4. Kocakulak, M., \& Butun, I. (2017, January). An overview of Wireless Sensor Networks towards internet of things. In 2017 IEEE 7th annual computing and communication workshop and conference (CCWC) (pp. 1-6). Ieee.

5. Reis, J. Z., \& Gonçalves, R. F. (2018, August). The role of internet of services (ios) on industry 4.0 through the service oriented architecture (soa). In IFIP International Conference on Advances in Production Management Systems (pp. 20-26). Springer, Cham.

6. Luckey, D., Fritz, H., Legatiuk, D., Dragos, K., \& Smarsly, K. (2020, August). Artificial intelligence techniques for smart city applications. In International Conference on Computing in Civil and Building Engineering (pp. 3-15). Springer, Cham.

7. Zhao, L., Wang, J., Liu, J., \& Kato, N. (2019). Optimal edge resource allocation in IoT-based smart cities. IEEE Network, 33(2), 30-35.

8. Guerrero-Ibáñez, J., Zeadally, S., \& Contreras-Castillo, J. (2018). Sensor technologies for intelligent transportation systems. Sensors, 18(4), 1212.

9. Guériau, M., Cugurullo, F., Acheampong, R. A., \& Dusparic, I. (2020). Shared Autonomous Mobility on Demand: A Learning-Based Approach and Its Performance in the Presence of Traffic Congestion. IEEE Intelligent Transportation Systems Magazine, 12(4), 208-218.

10. Ruta, K., Rakus, D., Ganzha, M., \& Paprzycki, M. (2020). AgentBased Traffic Obstacles Information System. Applied Soft Computing and Communication Networks: Proceedings of ACN 2019, 125, 81.

11. Tong, Y., Chen, L., \& Shahabi, C. (2017). Spatial crowdsourcing: Challenges, techniques, and applications. Proceedings of the VLDB Endowment, 10(12), 1988-1991.

12. Talukder, M. Z., Towqir, S. S., Remon, A. R., \& Zaman, H. U. (2017, July). An IoT based automated traffic control system with real-time update capability. In 2017 8th International Conference on Computing, Communication and Networking Technologies (ICCCNT) (pp. 1-6). IEEE. 
13. Rahaman, I., Reza, M. F., Hasib, M. H. H., Hossain, M. I., Hossain, S. A., \& Sarkar, P. K. (2019, July). A low cost intelligent multi wireless sensor network perspective on real time traffic surveillance. In 2019 International Conference on Computer, Communication, Chemical, Materials and Electronic Engineering (IC4ME2) (pp. 1-4). IEEE.

14. Zantalis, F., Koulouras, G., Karabetsos, S., \& Kandris, D. (2019). A review of machine learning and IoT in smart transportation. Future Internet, 11(4), 94.

15. Guerrero-Ibáñez, J., Zeadally, S., \& Contreras-Castillo, J. (2018). Sensor technologies for intelligent transportation systems. Sensors, 18(4), 1212.

16. Yu, Y., Li, Y., Xia, T., Deng, H., Bao, L., \& Li, W. (2017). Organizational mode innovation and credit supervision in road freight transportation under smart mobile devices applications services. Transportation Research Procedia, 25, 762-771.

Elrawy, M. F., Awad, A. I., \& Hamed, H. F. (2018). Intrusion detection systems for IoT-based smart environments: a survey. 